\title{
2-Particle Asymptotic Completeness and Bound States in Weakly Coupled Quantum Field Theories
}

\author{
J. Bros and D. Iagolnitzer
}

Service de Physique Théorique ${ }^{\star}$ de Saclay, F-91191 Gif-sur-Yvette Cedex, France

\begin{abstract}
Rigorous results on poles of the 2- and 4-point functions, which yield 2-particle asymptotic completeness and give information on the presence or absence of 2-particle bound states and resonances, are presented for weakly coupled even and non-even-field theories with mass gap in space-time dimension $d=2,3$ (and for related hypothetical theories in dimension 4). Methods used are more convenient and more general than those used previously (with more limited results) for $P(\varphi)_{2}$ theories.
\end{abstract}

\section{Contents}

1. Introduction . . . . . . . . . . . . . . . . . . . . . . . . . . . 331

2. Summary of Methods and Results . . . . . . . . . . . . . . . . . . . 333

3. Away from the 2-Particle Threshold (Even Theories). . . . . . . . . . . . . 336

4. The Neighborhood of the 2-Particle Threshold (Even Theories). . . . . . . . . 337

4.1. Preliminary Definitions and Results . . . . . . . . . . . . . . . . . 337

4.2. Applications to Theories in Dimension 4,2,3 . . . . . . . . . . . . . . 339

5. Non-Even Theories . . . . . . . . . . . . . . . . . . . . . . . . . 342

5.1. Preliminaries and Results Away from the 2-Particle Threshold . . . . . . . 342

5.2. The Neighborhood of the 2-Particle Threshold (First Method) . . . . . . . . 343

5.3. The Modified or Direct Bethe-Salpeter Kernel and Related Results. . . . . . 345

5.4. Application to $\lambda P(\varphi)_{2},\left(\lambda \varphi^{4}+\lambda^{\prime} \varphi^{3}\right)_{3}$ and Related Models . . . . . . . . . 347

Appendix 1. Results on 2-Particle Convolution . . . . . . . . . . . . . . . 347

Appendix 2. Technical Complements (Non-Even Theories) . . . . . . . . . . . 350

References. . . . . . . . . . . . . . . . . . . . . . . . . . . . 351

\section{Introduction}

Results on poles of the momentum-space 2- and 4-point functions, relevant in the study of 2-particle asymptotic completeness, bound states and resonances have been obtained in the second part of the seventies for weakly coupled even $P(\varphi)_{2}$ models [GJS, SZ, DE1, 2] and in a more limited way [K1, GJ] for non-even $P(\varphi)_{2}$

\footnotetext{
* Laboratoire de l'Institut de Recherche Fondamentale du Commissariat à l'Energie Atomique
} 
or related models (such as $\varphi_{2}^{4}$ with strong external field [K 1] or in low temperature phases $[\mathrm{K} 2, \mathrm{Im}])$. They have confirmed in particular the existence, expected in dimension 2 from heuristic arguments, of a pole close to the 2-particle threshold corresponding to a 2-particle bound or antibound state (depending on $P$ ). In these works, the basic ingredients are "irreducibility" and boundedness properties of the "2-particle irreducible" (2p.i.) Bethe-Salpeter kernel $B$ and, for non-even theories, of $2 \rightarrow 1,1 \rightarrow 2,1 \rightarrow 12$ p.i. kernels, established by the methods of [S] (and following also from those of [IM1]). These properties are: analyticity in complex energymomentum space in strips $\sum_{\varepsilon}$ around euclidean space going up to $s=(3 m)^{2}-\varepsilon$ in Minkowski space, or $(4 m)^{2}-\varepsilon$ in even theories, where $m$ is the bare mass and $s$ is the squared energy of the channel, bounds in cst $|\lambda|$, where $\lambda$ is the coupling, and further bounds in higher powers of $\lambda$ after lowest order contributions have been extracted. (These lower order contributions are shown to coincide with those of perturbation theory.) However, the methods used in these past works are not satisfactory under several respects. First, they do not exhibit in a simple way the complete meromorphic structure of the relevant Green functions near the twoparticle threshold and do not give a clear account of the roles played respectively by the small-coupling character of the models and by the threshold geometrical situation; moreover, they do not allow easy calculations of relevant quantities (positions of the poles, residues). On the other hand, the results obtained are only partial for non-even $P(\varphi)_{2}$ (or related) models. Finally and more important, the methods are specifically restricted, as they stand, to $P(\varphi)_{2}$ (or related) models, namely scalar models in dimension 2 .

More recently, a number of new models of interest have been introduced in constructive theory, such as $\left(\lambda \varphi^{4}+\lambda^{\prime} \varphi^{3}\right)_{3}$ in space-time dimension 3 , and [FMRS, GK] the renormalizable, but not super-renormalizable, asymptotically free massive Gross-Neveu model (GN) in dimension 2 (which involves spin and colour indices). Moreover, suitable BS or BS-type kernels have also been defined [IM2] in these theories: the BS kernel $B$, linked in $P(\varphi)_{2}$ models to the (connected, amputated) 4-point function $F$ by the BS equation

$$
F=B+B o F=B+F_{O} B
$$

where $o$ denotes Feynman-type convolution with 2-point functions $\mathrm{H}_{2}$ on internal lines, is replaced, in e.g. even theories like the $\mathrm{GN}$ model, by a kernel $B_{M}$ ( $M$ fixed) linked to $F$ via a regularized version of (1) (introduced in the general QFT framework in $[\mathrm{B} 1, \mathrm{BL}])$ where $o$ is replaced by an operation $o_{(M)}$ including ultraviolet cut-off factors (so as to avoid ultraviolet problems). Starting from the properties of the 2 p.i. kernels and related properties of the 2-point function $\mathrm{H}_{2}$, the analysis of [B 1, BL by BI 1], which is independent of the model, the dimension $d$ of spacetime or the coupling, then shows that $F$ is analytic in a 2-sheeted ( $d$ even) or multisheeted ( $d$ odd) domain around the 2-particle threshold $s=4 \mu^{2}$, where $\mu>0$ is the basic physical mass defined by the pole of $H_{2}$ [closed to $m$ at small $\lambda$ in $P(\varphi)_{2}$ models], apart from possible poles in $s$ (zeroes of denominators of Fredholm-type solutions) to be interpreted as 2-particle bound states in the physical sheet, and resonances in the non-physical sheet(s) (or "antibound" states at $s$ real in the second sheet). The analysis of [B 1, BL] yields moreover 2-particle asymptotic completeness in the region $(2 \mu)^{2}<s<(3 \mu)^{2}-\varepsilon$ or $(4 \mu)^{2}-\varepsilon$ in an even theory, with respect to particles of mass $\mu$, provided there is no pole of $F$ in that 
region (i.e. no bound states embedded in the continuum). It is based on methods that take into account explicitly the geometrical situation, namely the pinching of the integration cycle of the operation $o$ by the pole singularities of the two 2-point functions.

The determination of possible poles (positions, etc. ...) cannot be treated by such a general analysis without additional information on the kernel $B_{M}($ or $B)$. It turns out, as we shall see, that if $B_{M}$ (or $B$ ) is uniformly bounded by a sufficiently small constant - as it can be proven to hold in all constructible 2 or 3-dimensional small coupling models with mass gap [IM1,2] - the meromorphic structure of the relevant Green functions around the two-particle physical region (threshold included) can be completely understood by algebraic methods using refined geometrical properties of (Feynman-type) two-particle convolution. These properties represent simple and convenient improvements on certain features of those presented and used in [BP] (for the study of Fredholm theory in complex space) based on the original analysis of $[\mathrm{L}]$ and $[\mathrm{Ph}]$. On the other hand, a further algebraic progress linked to our geometrical approach can be performed for the treatment of non-even theories (determinations of poles etc....). It removes in particular the cumbersome problem of spurious poles that appear in the standard methods using one-particle irreducible functions.

Two articles will be devoted to the presentation of our methods and results (under various aspects), each one being essentially self-contained.

In the present article, stress is laid upon the " $U$ kernel"-method inspired from [BI1] and more specifically [Ia], which leads to the simplest algebraic description. We show that it allows one to reobtain and complete previous results on poles for weakly coupled $P(\varphi)_{2}$-models, and to extend the analysis to other even or noneven theories in dimensions 2 and 3 (and to related hypothetical theories in dimension 4). A summary of methods and results is given in Sect. 2.

The other article [BI2] will give complements on the geometrical background (making the relevant properties of $[\mathrm{Ph}]$ and $[\mathrm{BP}]$ used in this article more easily visualized) and will present an alternative algebraic method (closer in some respect to the method of [DE2]) based on appropriate decompositions of Fredholm resolvents in complex space. This method will be also applied to constructing (nonlocal) potentials associated with BS type kernels $B_{M}$ (or $B$ ) and to obtaining a comprehensive viewpoint on (2- and 3-dimensional) threshold effects in both QFT and potential formulations of scattering theory, in the spirit of [B2].

In these papers, we have left aside for conciseness related topics, partly treated previously for $P(\varphi)_{2}$ models, such as the determination of fields that generate 2-particle bound states $\left(: \varphi(x)^{2}:\right)$, the $C^{\infty}$ or analytic dependence in $\lambda$ or also Borel summability properties [EE] of various quantities (bound-state mass, 2-particle $S$ matrix ,...) or the introduction of the $S$-matrix for processes involving bound states. All results can be reobtained, improved and extended.

\section{Summary of Methods and Results}

Throughout this paper, we restrict our attention to regions limited to $s=(3 \mu)^{2}-\varepsilon$, or $(4 \mu)^{2}-\varepsilon$ in even theories; $\varepsilon>0$ is fixed (it can be chosen arbitrarily small but corresponding constraints follow on the maximal possible values of the coupling). 
We first treat even theories in Sects. 3 and 4 (in any spacetime dimension $d$ ). Non-even theories are treated in Sect. 5. The crucial tool concerning two-particle convolution, stated as Lemma 1 in Sect. 4, is proved in Appendix 1.

Even theories. The relevant Green function for even theories [at $\left.s<(4 \mu)^{2}-\varepsilon\right]$ is the 4-point function $F$. It is first shown in Sect. $3^{1}$ that, at small coupling, possible poles of $F$, if they exist, lie either "far away" in the unphysical sheet(s) or close to the 2-particle threshold $s=4 \mu^{2}$. This is due to convergence properties, at small coupling, of "the Neumann series $F=B+B o B+B o B o B+\ldots$ (or $F=B_{M}$ $+B_{M} o_{(M)} B_{M}+\ldots$ ), where terms $B o \ldots o B\left(\right.$ or $\left.B_{M} O_{(M)} B_{M} \ldots\right)$ are defined by analytic continuation away from euclidean space, in a 2 -sheeted (or multisheeted) domain around $s=4 \mu^{2}$ (the analyticity of $B$ in a strip $\Sigma_{\varepsilon}$ around euclidean space being taken into account, as the basic starting point): to that purpose, locally distorted integration contours (initially euclidean space) over the internal relative energymomentum are introduced following $[\mathrm{B} 1, \mathrm{BL}]$ so as to avoid the pole singularities (at the physical mass) of 2-point functions involved in $o\left(\right.$ or $\left.o_{(M)}\right)$. Problems close to $s=4 \mu^{2}$ are due to the pinching of this contour between these poles as $s \rightarrow 4 \mu^{2}$.

Further investigations are then restricted to values of $s$ in a given complex neighborhood $V$ of $s=4 \mu^{2}$. At $d=4$, it will be established in Sect. 4 that, at small coupling, $F$ has no pole for $s$ in $V$ either in the first or second sheet ${ }^{1}$. In dimensions 2 and 3 the situation is different in view of specific kinematical factors, as well known from heuristic arguments. The local structure of $F$ for $s$ in $V$, in its two sheets ( $d$ even) or in a finite number of sheets ( $d$ odd), including its possible poles and residues, and its behaviour at threshold, is then exhibited in terms of the kernel $U$ defined in [Ia] from the BS kernel $B$ (or $B_{M}$ ) by the equation:

$$
U=B+U \nabla B
$$

(leaving from now on $M$ implicit: $U$ is in fact independent of the choice of $M$ ), where:

$$
\nabla=o-g(s) * .
$$

* denotes on-mass-shell convolution and $g=1 / 2$ if $d$ is even, $g=\frac{i}{2 \pi} \ln \sigma, \sigma=4 \mu^{2}-s$, if $d$ is odd: in either parity case, $\nabla$ is shown to be "regular," i.e. to preserve analyticity for $s$ in $V$ [in other words, $g(s) *$ extracts the singular part of $o$ at $s=4 \mu^{2}$ ] so that (2) defines $U$ at small coupling as the locally analytic, convergent sum of the Neumann series $B+B \nabla B+B \nabla B \nabla B+\ldots(U$ is equal to $B$ at lowest order in the coupling). In view of (2), the BS equation (1) is transformed into the equation:

$$
\begin{aligned}
F= & U+g(s) U * F \\
& =U+g(s) F * U,
\end{aligned}
$$

i.e. $U$ coincides on shell with the " $K$-matrix" $[\mathrm{H}, \mathrm{Z}, \mathrm{POLE}]$ at $d$ even or its substitute introduced in [BI] at $d$ odd, defined in these works in terms of $F$ by Eq. (3) (which can also be used to define $U$ off shell: see [Ia]).

Remark. Local analyticity of $U$ is derived in these axiomatic, $S$-matrix or field theory approaches from 2-particle unitarity or asymptotic completeness (AC)

\footnotetext{
${ }^{1}$ These results, which are essentially contained in Proposition 3 of [BP], are given here a more condensed derivation, in a form which is better adapted to the problem under study
} 
(and some further technical assumptions) via Eq. (3). In the present work, concerned with constructible QFT models, 2-particle AC is not assumed but is part of the results to be established, so that the local analyticity of $U$ has been derived instead from the analyticity of $B$ in $\Sigma_{\varepsilon}$ via Eq. (2).

At $d=2$, the on-mass-shell convolution $*$ reduces to a mere multiplication. Equation (3) can therefore be solved for $F$ by elementary algebra and yields, with

$$
\begin{gathered}
k=p_{1}+p_{2}=p_{3}+p_{4}, z=\frac{p_{1}-p_{2}}{2}, z^{\prime}=\frac{p_{3}-p_{4}}{2}: \\
F\left(k, z, z^{\prime}\right)=U\left(k, z, z^{\prime}\right)+\frac{U_{1}(k, z)_{1} U\left(k, z^{\prime}\right)}{a(s)-u(s)},
\end{gathered}
$$

where $U_{\mid}$and $U$ are the well defined values of $U$ obtained for values of $z^{\prime}$, respectively of $z$, such that $p_{3}, p_{4}$, respectively $p_{1}, p_{2}$, are on-shell, $u$ is the full onshell restriction of $U$ and $a(s)$ is a kinematical factor [arising from $g(s)$ and $*$ ] behaving like $\operatorname{cst} \sigma^{1 / 2}$ as $\sigma \rightarrow 0, \sigma=4 \mu^{2}-s$. This factor is at the origin of one and only one pole for $s$ in $V$ [namely the zero of $a(s)-u(s)]$ at $s=\mu_{B}^{2}<4 \mu^{2}$, however small $\lambda$ is, either in the physical sheet or in the second sheet depending on the sign of $u$ near $\sigma=0$ : in e.g. a $\lambda P(\varphi)_{2}$ theory with a non-zero $\pm \lambda \varphi^{4}$ term, $u=\mp \operatorname{cst} \lambda$, as $B$, at lowest order in $\lambda$, and one has respectively an antibound or bound state, with $\left|\mu_{B}-2 \mu\right|=\operatorname{cst}|\lambda|^{2}+O\left(\lambda^{3}\right)$. One can similarly obtain from the equation $a(s, \lambda)$ $-u(s, \lambda)=0$ asymptotic expansions of $\mu_{B}(\lambda)$ to higher orders in $\lambda$, starting from those on $B$ in terms of Feynman integrals and using those on $u$ that follow from the Neumann series of Eq. (2). [For related results, e.g. on the residue of the (bound state)-(bound state) 2-point function at its pole at $s=\mu_{B}^{2}$, see Sect. 4.]

Spinorial models like the GN model are treated in the same way, with only minor changes (see Sect. 4).

At $d=3$, the results are similar $F$ is decomposed as $\stackrel{0}{F}+\stackrel{1}{F}$ where $\stackrel{0}{F}$ is the " $l=0$ partial wave component" of $F$ and $\stackrel{1}{F}$ is its complement, shown to be locally bounded. $\stackrel{0}{F}$ is again given by formula (4) with $U$ replaced by $\stackrel{0}{U}$. The factor $a(s)$ now behaves like cst $/ \ln \sigma$ as $\sigma \rightarrow 0$ : hence, there is in general (at small coupling) either no pole for $s$ in $V$ or one pole in the physical sheet at $s=\mu_{B}^{2}<4 \mu^{2}$, with $\left|\mu_{B}-2 \mu\right| \approx \operatorname{cst} e^{-\operatorname{cst} / \lambda}$. There is no pole for $\varphi_{3}^{4}$. There will be one pole in the non-even $\left(\lambda \varphi^{4}+\lambda^{\prime} \varphi^{3}\right)_{3}$ models for some values of $\lambda, \lambda^{\prime}$.

Non even theories. The 4-point function $F$ satisfies the equation:

$$
F=F_{1}+=(1)-(k, z) H_{2}(k)-(1)=\left(k, z^{\prime}\right),
$$

where $F_{1} \equiv=(1)=$ is the (connected, amputated) 1-p.i. function, linked to the 2-p.i. BS kernel $B$ (or $B_{M}$ ) by the BS equation, and =(1)- $-(1)=$ are similar 3-point 1-p.i. kernels satisfying e.g.

$$
=(1-==2-+=(1)=(2),
$$

where $=2$ - is the 2-p.i. kernel and the last term is the Feynman-type convolution $F_{1} O=$ (2)- On the other hand, the kernel-(1)-defined as $-1 / H_{2}$, equal e.g. in $P(\varphi)_{2}$ to $s-m^{2}+I_{1}$, where $I_{1}$ is the actual $1 \rightarrow 1$ 1-p.i. function, satisfies:

$$
-(1)=-(2)+-(1)=(2) \text {, }
$$


where -(2)- $=s-m^{2}+I_{2}$ in $P(\varphi)_{2}, I_{2}$ being the actual 2-p.i. kernel. Equation (7) follows from the similar relation between $I_{1}$ and $I_{2}$ :

$$
I_{1}=I_{2}+\text { - (1)- (2) }
$$

From (5)-(7) and known properties ([S], [IM 1,2]) of the 2-p.i. kernels, the analogue of the result of Sect. 3 is again established (Sect. 5.1). Note that $F$ has a pole, as $H_{2}$, at $s=\mu^{2}$ and that $H_{2}$ is now expected to have itself poles, if $F$ has, in the neighborhood $V$ of $s=4 \mu^{2}$.

Two methods are given to determine the structure of $F$ and $H_{2}$ for $s$ in $V$. The first one (Sect. 5.2) starts, as [K1], from (5)-(7) and makes use of results on $F_{1}$ which are obtained in the same way as in even theories from the BS equation and the properties of $B$ : the simple and more complete results obtained on $F_{1}$ (in particular on its behaviour at threshold) and those on $\nabla$ allow an easy derivation of complete results on $\mathrm{F}$ and $\mathrm{H}_{2}$, in contrast to [K1] where only partial results (at $s<4 \mu^{2}$ ) were obtained.

An alternative method (Sect. 5.3) makes use of the "modified" or "direct" BS kernel $G$ linked to $F$ itself by the BS equation. $G$ was defined in [B 1$]$ in terms of $F$ by this equation, and its analyticity properties were then derived from 2-particle $\mathrm{AC}$ (and further conditions). In this work, where 2-particle AC is not assumed, $G$ will be defined instead in terms of the basic 2-p.i. kernels by the relation:

$$
G==(2)=-\frac{=(2)-(k, 2)-(2)=\left(k, z^{\prime}\right)}{-2} \text {. }
$$

This relation, which is new to our knowledge, yields properties of $G$ similar to those of $B$ at small coupling, at least away from a neighborhood of $s=\mu^{2}$ (where a zero of -(2)-introduces a pole close to $\mu^{2}$ ). The analysis of $F$ for $s$ in $V$, where $G$ is analytic, is then completely analogous to that presented in Sect. 4 for even theories, with $B$ replaced by $\mathrm{G}$. Results on $\mathrm{H}_{2}$ also follow.

As a matter of fact, both methods are closely related. As expected from general arguments (see Sect. 5.1), possible poles of $F_{1}$ and of $F$ (and $H_{2}$ ) are different. The cancellation of the pole of $F_{1}$ with a corresponding pole in the second term in the right-hand side of (5), and the possible appearance of a different pole, are easily seen in the first method. The second one has the conceptual advantage of avoiding this problem from the outset by eliminating $F_{1}$. (Note, however, that $G$ introduces spurious poles elsewhere as already mentioned.)

\section{Away from the 2-Particle Threshold (Even Theories)}

We consider any theory in dimension $d=2,3$, or 4 , depending on a coupling parameter $\lambda$, in which

(i) either [as in $\lambda P(\varphi)_{2}$ or $\left.\lambda \varphi_{3}^{4}\right]$, a BS kernel $B$ linked to $F$ by Eq. (1) is defined, with $B\left(k, z, z^{\prime}\right)$ and $\left(k^{2}-\mu^{2}\right) H_{2}(k)$ analytic in respective (complex) domains of the form $\left(k, z, z^{\prime}\right) \in \Delta \times \mathscr{D} \times \mathscr{D}$ and $k \in \Delta^{\prime}, \Delta, \mathscr{D}, \Delta^{\prime}$ as in $[\mathrm{BL}, \mathrm{SZ}]$, in particular $\left|\operatorname{Re} k_{0}\right|$ $<4 \mu-\varepsilon, \varepsilon>0$ fixed for $k$ in $\Delta$; here $k$ is taken real in Minkowski space and purely imaginary in euclidean space so that $s=k_{0}^{2}$ if $k$ is of the form $\left(k_{0}, \mathbf{0}\right) .|B|$ and $\left|\left(k^{2}-\mu^{2}\right) H_{2}\right|$ are moreover bounded in their domains by $C_{1}|\lambda|$ and by $C_{2}$ respectively; $k^{2}=k_{0}^{2}-\mathbf{k}^{2}$. 
(ii) or, as in the GN model or possibly in hypothetical theories at $d=4$, there exist quantities $B_{M}, H_{2}^{(M)}, M$ fixed, linked to $F$ by the relation

$$
F=B_{M}+F o_{M} B_{M},
$$

$B_{M}$ and $H_{2}^{(M)}$ having the analyticity properties indicated in (i) for $B$ and $H_{2}, H_{2}^{(M)}$ having the same pole at $s=\mu^{2}$ and same residue there as $H_{2} \cdot\left|B_{M}\right|$ is bounded by $C_{1}|\lambda|$ and $\left\{\left(s-\mu^{2}\right) H_{2}^{(M)} \mid\right.$, e.g. (as shown in [IM2] for the GN model) by $C_{2} e^{- \text {cst }\left|k^{2}\right|}$.

We then state:

Proposition 1. Given any bounded (compact) region D in the 2-sheeted (d even) or multi-sheeted ( $d$ odd) domain of $F$ whose distance to $s=4 \mu^{2}$ is strictly positive, $\exists \lambda_{0}>0$ such that $F$ has no pole in $D$ if $|\lambda|<\lambda_{0}$.

Proof. The terms $B o \ldots o B$ ( $n$ factors $B$ ) of the Neumann series of $F$ in terms of $B$, defined away from euclidean space by analytic continuation as indicated in Sect. 1, satisfy in $D$ bounds of the form:

$$
\left|(B o \ldots o B)\left(k, z, z^{\prime}\right)\right|<C\left(C_{1}^{\prime}|\lambda|\right)^{n},
$$

where $C, C_{1}^{\prime}$ depend on $C_{1}$ and on $D$ but not on $\lambda$. (The dependence on $D$ is due to the fact that the contour must be more and more distorted as $D$ becomes larger and larger and that it must approach more and more the pole singularities of 2-point functions as the distance of $D$ to $s=4 \mu^{2}$ tends to zero.)

The Neumann series is absolutely convergent, and $F$ is bounded (thus has no pole) at small enough $\lambda$. Q.E.D.

\section{The Neighborhood of the 2-Particle Threshold (Even Theories)}

4.1. Preliminary Definitions and Results. We restrict our attention from now on to values of $s$ in $V$ (see Sect. 1). We start for simplicity with scalar models. (Easy modifications for spinorial models like the GN model are left to the discussion of this model in Sect. 4.2.)

The operation $*_{+}$will denote, in the region $s$ real, $s>4 \mu^{2}$ obtained from the directions $\operatorname{Im} s>0$ of the physical sheet, on mass-shell convolution over two internal energy-momenta: more precisely given two kernels $A\left(k, z, z^{\prime}\right), B\left(k, z, z^{\prime}\right)$,

$$
A *{ }_{+} B\left(k, z, z^{\prime}\right)=\int A(k, z, \zeta) B\left(k, \zeta, z^{\prime}\right) Z^{2} \delta\left(\left(\frac{k}{2}+\zeta\right)^{2}-\mu^{2}\right) \delta\left(\left(\frac{k}{2}-\zeta\right)^{2}-\mu^{2}\right) d \zeta
$$

with here $k$, e.g. of the form $\left(k_{0}, \mathbf{0}\right), k_{0}$ real; $Z$ is the residue of $H_{2}$ at its pole. Equation (11) can be equally written as:

$$
(A *+B)\left(k, z, z^{\prime}\right)=\frac{Z^{2}}{2 \sqrt{s}}(-\sigma)^{\frac{d-3}{2}} \int A(k, z, \zeta(s, \Omega)) B\left(k, \zeta(s, \Omega), z^{\prime}\right) d \Omega,
$$

where $\Omega$ is a variable varying on the real unit $(d-2)$-dimensional sphere (reducing to an angle $\theta$ at $d=3$, the integral reducing moreover to a sum over two points at $d=2)$, and if e.g. $k$ is of the form $\left(k_{0}, \mathbf{0}\right), s=k_{0}^{2}, \zeta_{0}(s, \Omega)=0, \zeta^{2}(s, \Omega)=-\sigma$. 
Formula $\left(11^{\prime}\right)$ allows us in turn to define $*$ by analytic continuation for real or complex values of $s$ in a one-sheeted ( $d$ odd) or 2-sheeted ( $d$ even) domain around $\sigma=0$, assuming here analyticity of $A$ and $B$ for $s$ in $V$.

The operation $o$ will denote, for values of $s$ in a 2-sheeted ( $d$ even) or infinitelysheeted $(d$ odd) domain constructed on $V$, Feynman-type convolution ("G-convolution" in the sense of $[\mathrm{B} 1, \mathrm{BL}]$ ) with the integration measure $\omega(k, \zeta) d \zeta, \omega(k, \zeta)=H_{2}\left(\frac{k}{2}+\zeta\right) H_{2}\left(\frac{k}{2}-\zeta\right)$, over the possibly locally distorted contour $\Gamma(k)$ already mentioned in Sect. 1 [B 1, BL].

The operation $\nabla$ is defined by Eq. $\left(2^{\prime}\right)(\nabla=o-g(\sigma) *)$.

We then state:

Lemma 1. Given two functions $A\left(k, z, z^{\prime}\right), B\left(k, z, z^{\prime}\right)$ analytic in $\Delta \times \mathscr{D} \times \mathscr{D} \cap\{s \in V\}$, and bounded in modulus by constants $\|A\|$ and $\|B\|$ respectively, $A \nabla B$ is analytic and bounded there in modulus by $C^{\prime}\|A\| \times\|B\|$, where $C^{\prime}$ is a given constant, independent of $\|A\|$ and $\|B\|$ (and depending only on the given bounds on $\left|\left(k^{2}-\mu^{2}\right) H_{2}\right|$ ).

The uniformity of $\nabla(A \nabla B$ uniform when one turns around $\sigma=0) \mid$ has already been explained in [Ia]. This and the more complete proof of Lemma 1 are given in Appendix 1.

Lemma 2. $\exists \lambda_{0}>0$ such that $\forall \lambda,|\lambda|<\lambda_{0}, E q$. (2):

$$
U=B+U \nabla B
$$

defines the kernel $U\left(k, z, z^{\prime}\right)$ as an analytic function in $\Delta \times \mathscr{D} \times \mathscr{D}, s \in V$, bounded by $C_{1}^{\prime}|\lambda|$, where $C_{1}^{\prime}$ depends only on the bounds on $|B|$ (in $C_{1}|\lambda|$ ) and of those on $\left|\left(k^{2}-\mu^{2}\right) H_{2}\right|$. Moreover the relations (3) (3')

$$
F=U+g(s) F * U=U+g(s) U * F
$$

as also

$$
F=U+g(s) U * U+g(s)^{2} U * F * U
$$

hold for $s$ in a 2-sheeted ( $d$ even) or multisheeted ( $d$ odd) domain constructed over $V \backslash\left\{s=4 \mu^{2}\right\}$.

Proof. This result is essentially contained in [Ia] except that the kernel $U$ was only shown there to be meromorphic and uniform around $\sigma=0$, but not analytic. We here complete the proof. From Lemma 1 and the bounds on $|B|$, the terms $|B \nabla B \ldots \nabla B|(n$ factors $B)$ are bounded by $C_{1}|\lambda|\left(C^{\prime} C_{1}|\lambda|\right)^{n-1}$. The Neumann series $U=B+B \nabla B+B \nabla B \nabla B+\ldots$ is thus absolutely convergent [for e.g. $\left.|\lambda|<1 /\left(2 C_{1} C^{\prime}\right)\right]$ and results on $U$ follow.

Equation (3) is obtained by writing e.g.

$$
F=B+B \circ F=(U-U \nabla B)+U \circ F-(U \nabla B) \circ F,
$$

and using

$$
(U \nabla B) \circ F=U \nabla(B \circ F)=U \nabla(F-B)=U \nabla F-U \nabla B .
$$

(The equality $B \nabla U=U \nabla B$ is straightforward.) 
Equation $\left(3^{\prime}\right)$ is obtained similarly and (12) follows by a repeated application of (3) and (3').

Remarks. 1) We note that (through an adaptation of Fredholm theory in complex space [BP] to the operator $\nabla$ ) Eq. (2) defines $U$ in terms of $B$ even in cases when the series $B+B \nabla B+\ldots$ would not be convergent: $U$ is in general uniform when one turns around $\sigma=0$, but might have poles in $s$.

2) Results above can be recovered formally by starting from the series $F=B$ $+B o B+B o B o B+\ldots$ and replacing $o$ by $(g(s) *)+\nabla$. A simple regrouping of terms gives

$$
\begin{aligned}
F & =\sum(B+B \nabla B+\ldots) g(s) *(B+B \nabla B+\ldots) g(s) * \ldots \\
& =\sum U(g(s) *) U(g(s) *) U \ldots,
\end{aligned}
$$

which is the Neumann series of $F$ in (3).

4.2. Applications to Theories in Dimension 4, 2, 3. We now draw conclusions in dimensions 4,2 , and 3 respectively.

$d=4$. Proposition 1 still holds when the restriction on the distance of $D$ to $s=4 \mu^{2}$ is removed. The series $\sum B o B \ldots o B$ remains in fact convergent at small coupling for $s$ in $V$ as can be seen by decomposing near $\sigma=0, o$ as $\nabla+\frac{1}{2} *$ and noting that the factor $\sigma^{(d-3) / 2}=\sigma^{1 / 2}$ generated by $*$ [see formula $\left.\left(11^{\prime}\right)\right]$ gives no problem (see also $[\mathrm{BP}]$, where a detailed derivation of bounds on the terms $|B o B \ldots o B|$ has been given in the proof of Proposition 3). Alternatively, the fact that $F$ is bounded (and thus has no pole) near $\sigma=0$ can be derived from the convergence of the Neumann series $U+g U * U+g^{2} U * U * U+\ldots$ of $F$ in terms of $U$ (due to the same reason).

$d=2$ [scalar models such as $P(\varphi)_{2}$ ]. The mass-shell is trivial at $d=2$ (the relation $p_{1}+p_{2}=k, p_{1}, p_{2}$ on shell, determines $\left.{ }^{2} p_{1}, p_{2}\right)$. Values of $F$ and $U$ when $p_{3}, p_{4}$ (respectively $p_{1}, p_{2}$ ) are on shell but not necessarily $p_{1}, p_{2}$ (respectively $p_{3}, p_{4}$ ) depend only on $s$ and $z$ (respectively $\left.s, z^{\prime}\right)$ and will be denoted $F_{\mid}(s, z)$ and $U_{\mid}(s, z)$ [respectively $\left.F\left(s, z^{\prime}\right),{ }_{\mid} U\left(s, z^{\prime}\right)\right]$. The full mass-shell restrictions of $F$ and $U$ depend only on $s$ and will be denoted $f(s)$ and $u(s)$.

The composition product $(*)$ involved in Eq. $\left(3^{\prime}\right)$ then reduces to:

$$
g(s)[F * U]\left(k, z, z^{\prime}\right)=\frac{1}{a(s)} F_{\mid}(k, z)_{\mid} U\left(k, z^{\prime}\right),
$$

where the factor $1 / a(s)$ is the product of $\frac{1}{2} Z^{2}$ with the kinematical factor generated by $*\left[\right.$ see $\left.(11),\left(11^{\prime}\right)\right]$, of the form $\operatorname{cst} /\left(\sqrt{s} \sigma^{1 / 2}\right)$.

One therefore obtains

Proposition 2. The following structural equations, in which $U, U_{1},{ }_{1} U$, and $u$ are analytic for $s\left(=4 \mu^{2}-\sigma\right)$ in $V$, hold, respectively on-shell and off-shell:

\footnotetext{
${ }^{2} \mathrm{Up}$ to the permutation $\left(p_{1}, p_{2}\right) \rightarrow\left(p_{2}, p_{1}\right)$; in fact, the mass shell reduces to a zero-dimensional sphere, i.e. a couple of points on which $U$, as well as $F$, takes the same value in view of the symmetry of these functions with respect to the two incoming and to the two outgoing field operators in the channel considered
} 
a)

b) $\quad F\left(k, z, z^{\prime}\right)=U\left(k, z, z^{\prime}\right)+\frac{U_{\mid}(k, z){ }_{\mid} U\left(k, z^{\prime}\right)}{a(s)-u(s)}$.

Proof. Equation (14) follows directly from Eqs. (3) and (13). Equation (15) follows in turn from Eq. (12), rewritten in the form:

$$
F=U+\frac{1}{a(s)} U_{1} \cdot{ }_{1} U+\frac{1}{a(s)^{2}} U_{1} \cdot f \cdot{ }_{1} U,
$$

where [in view of $(14)]$ :

$$
f=\frac{a u}{a-u}
$$

These relations completely characterize the local structure of $f$ and $F$, for $s$ in $V$, including possible poles (=zeroes of $a-u$ ), their residues and their behaviour at threshold: $F$ remains bounded as $\sigma \rightarrow 0$ (for any given, small, $\lambda$ ) if $u$ is different from zero at $\sigma=0$ (as is the case at small $\lambda \neq 0$ : see below) and $F_{\mid},{ }_{\mid} F$ or $f$ tend to zero as $a$, i.e. like $\sigma^{1 / 2}$, as $\sigma \rightarrow 0$. q.e.d.

$U$ is equal at first order in $\lambda$ to $B$. On the other hand, $B$ is known (by the methods of [GJS], [SZ] or of [IM1]) to be equal to a first known term in $\lambda$ (theories with a $\varphi^{4}$ term) or $\lambda^{2}$ (theories without $\varphi^{4}$ term) plus a remainder bounded by higher powers of $\lambda$ (times a constant). Since $a(s)=(\operatorname{cst} \sqrt{s}) \sigma^{1 / 2}(s$ near $4 \mu^{2}, \sigma$ near zero), all results already known follow easily: at small $\lambda$ and for $s$ in $V$, one and only one pole at $s=\mu_{B}^{2}, \mu_{B}<2 \mu,\left|\mu_{B}-2 \mu\right|=\operatorname{cst} \lambda^{2}+O\left(\lambda^{3}\right)$ in theories with $\mathrm{a} \pm \lambda \varphi^{4}$ term in the interaction (see Sect. 1) either in the physical sheet $\left(-\lambda \varphi^{4}\right)$ or in the second sheet $\left(+\lambda \varphi^{4}\right)$. From Eq. (15), one also determines the residue $r(\lambda)$ at its pole $s=\mu_{B}^{2}$ of the (bound state)-(bound state) 2-point-function: $r(\lambda)$ $=\lim _{s \rightarrow \mu_{B}^{2}}\left(-s+\mu_{B}^{2}\right) \bullet$ F) $\sim \operatorname{cst} \lambda$ (using $U_{1} \sim \operatorname{cst} \lambda, \bigcirc \sim \operatorname{cst} \sigma^{-1 / 2}$, hence $\sim \operatorname{cst} / \lambda$ at the pole). The case with no $\varphi^{4}$ term is also treated easily (one two-particle bound state. The lowest order contribution to $B$ is in $\lambda^{2}$, with a sign that gives a pole in the physical sheet, again at $s=\mu_{B}^{2}<4 \mu^{2}$ : see e.g. [DE2]. Note that in this case $\left|\mu_{B}-2 \mu\right|$ $=\operatorname{cst} \lambda^{4}+O\left(\lambda^{5}\right)$ as exhibited by Eq. (17)).

$d=2$ (spinorial case: GN model). All equations involve now matrices (whose coefficients are functions of $\left.k, z, z^{\prime}, \ldots\right)$, e.g. for the $\mathrm{GN}$ model $4 \times 4$ matrices, some of which arise from the $2 \times 2$ matrices associated with 2-point functions, or in the case of Eq. (14) of their residues at the pole $k^{2}=\mu^{2}$.

The results are, however, essentially unchanged.

Poles of $F$ are now the zeroes of $\operatorname{det}(a(s) \mathbb{1}-m(s) u(s))$, where $m(s)$ is the $4 \times 4$ matrix obtained from the $2 \times 2$ residue matrices. The matrix $m$ has elements that are almost all equal to zero at threshold $\sigma=0$ so that conclusions at small coupling are easy.

The detailed analysis requires, however, the consideration of different channels ( $\psi \psi$ or $\psi \bar{\psi}$, spin and colour indices) and is thus omitted for conciseness. 
$d=3\left(\varphi_{3}^{4}\right)$. It is useful to introduce the decompositions $F=\stackrel{0}{F}+\stackrel{1}{F}, B=\stackrel{0}{B}+\stackrel{1}{B}$, $U=\stackrel{0}{U}+\stackrel{1}{U}$ by the following procedure. In view of invariance properties (namely Lorentz invariance and symmetry of the four-point interaction considered with respect to the two incoming and to the two outgoing field operators), $F, G, U$ are analytic functions of the variables $s=k^{2}, z_{0}, \mathbf{z}^{2}, z_{0}^{\prime}, \mathbf{z}^{\prime 2}$, and $\cos \theta, \theta$ being the angle between $\mathbf{z}, \mathbf{z}^{\prime}$ (i.e. the "scattering angle" of the $s$-channel). Then one puts $\stackrel{0}{F}=\frac{1}{2 \pi} \int F d \theta$ (at fixed values of $\left.s, z_{0}, \mathbf{z}^{2}, z_{0}^{\prime}, \mathbf{z}^{\prime 2}\right)$ and $\stackrel{1}{F} \equiv F-\stackrel{0}{F} ; \stackrel{0}{B}, \stackrel{0}{U}, \stackrel{1}{B}, \stackrel{1}{U}$ are defined similarly.

We now notice that the operation $\circ$ can be represented by integration cycles $\Gamma(k)$ that are invariant under the rotation group of the $\mathbf{z}$-plane; o therefore decomposes into a convolution in the angular variable of this rotation group and integration in the "mass variables" $\left(z_{0},\left[\mathbf{z}^{2}\right]^{1 / 2}\right)$ which play the same role as the variables $\left(z_{0}, z_{1}\right)$ of the two-dimensional operation $\circ$. Similar properties hold obviously for the operation $\nabla$, since $*$ is a pure angular convolution on the mass shell. It therefore follows from the definition of $\stackrel{0}{B}$ together with the relation $\int \stackrel{1}{B} d \theta=0$, that one has the orthogonality relations $\stackrel{0}{B} \circ \stackrel{1}{B}=\stackrel{0}{B} * \stackrel{1}{B}=\stackrel{0}{B} \nabla \stackrel{1}{B}=0$ which imply the decoupling of Eqs. (1), (2), (3), namely

$$
\begin{aligned}
& \stackrel{0}{F}=\stackrel{0}{B}+\stackrel{0}{F} \circ \stackrel{0}{B}, \\
& \stackrel{0}{U}=\stackrel{0}{B}+\stackrel{0}{U} \nabla \stackrel{0}{B}, \\
& \stackrel{0}{F}=\stackrel{0}{=}+\frac{i}{2 \pi} \ln \sigma \stackrel{0}{\sigma} * \stackrel{0}{U}, \\
& \stackrel{1}{F}=\stackrel{1}{B}+\stackrel{1}{F} \circ \stackrel{1}{B}, \\
& \stackrel{1}{U}=\stackrel{1}{B}+\stackrel{1}{U} \nabla \stackrel{1}{B}, \\
& \stackrel{1}{F}=\stackrel{1}{U}+\frac{i}{2 \pi} \ln \stackrel{1}{F} * \stackrel{1}{U} .
\end{aligned}
$$

$\stackrel{0}{F}, \stackrel{0}{B}, \stackrel{0}{U}$ do not depend on $\theta$ and can thus be treated in the same way as at $d=2$. The only difference is that, in the factor $a(s), \sigma^{1 / 2}$ is replaced by $-\frac{2 \pi}{\ln \sigma}$ (real at $\sigma>0$, i.e. $s<4 \mu^{2}$, in the physical sheet): the factor $\sigma^{-1 / 2}$ generated by $*$ at $d=2$ is absent at $d=3(d-3=0)$ but the factor $\ln \sigma$ comes from $g(s)$. Still denoting (by an abuse of notation) by $a(s)$ this new factor, Eqs. (14)-(16) hold again with $F, U$ replaced by $\stackrel{0}{F} \stackrel{0}{U}$.

Let us now show that, at small coupling the solution $\stackrel{1}{F}$ of Eqs. (21), (23) is necessarily bounded (although ramified in the Riemann surface of $\ln \sigma$ at $\sigma=0$ ) for $s$ in $V$, so that the possible pole structure of $F=\stackrel{0}{F}+\stackrel{1}{F}$ is entirely given by $\stackrel{0}{F}$.

We first notice that, since the mass shell reduces to the single point $z_{0}^{\prime}=0, \mathbf{z}^{\prime}=0$ at $\sigma=0$, the Lorentz invariant function $\left.B(k, z, 0)\right|_{\sigma=0}$ is independent of $\theta$ and therefore $\left.\stackrel{1}{B}(k, z, 0)\right|_{\sigma=0}$ vanishes identically. By applying Schwarz' lemma (in the 
variable $\sigma$ ) to the analytic function $\stackrel{1}{B_{1}}=\left.\stackrel{1}{B}\left(k, z, z^{\prime}\right)\right|_{z_{0}^{\prime}=0, \mathbf{z}_{0}^{\prime 2}=-\sigma}$, for $s$ in $V$, one then obtains (since $B$ and therefore $B$ are uniformly bounded by $\operatorname{cst} \times|\lambda|$ ) $\sup \left|B_{1}\right|$ $<\operatorname{cst} \times|\sigma||\lambda|$, and therefore [in view of Lemma 2 applied to Eq. (22)] $\sup \left|U_{1}\right|$ $<\operatorname{cst} \times|\sigma||\lambda|$. We now use this estimate in Eq. (23), first taken on the mass shell in order to obtain that $\sup |f|<\operatorname{cst} \times|\sigma||\lambda|$, and then off-shell under the form similar to $(16)$,

$$
\stackrel{1}{F}=\stackrel{1}{U}+\frac{i}{2 \pi} \ln \sigma \stackrel{1}{U}, \stackrel{1}{U}^{U}-\frac{1}{(2 \pi)^{2}}(\ln \sigma)^{2} \stackrel{1}{U_{1}} * \stackrel{1}{f} * \stackrel{1}{U}^{U}
$$

to obtain that $\stackrel{1}{F}$ is bounded (like $\stackrel{1}{U}$ ) for $s$ in $V$. We note that the bounds obtained on $|\stackrel{1}{f}|$ and $|\stackrel{1}{F}|$ are valid in any finite number of sheets of the Riemann surface of $\ln \sigma$, provided the value of $\lambda$ is chosen sufficiently small.

We can therefore state

Proposition 3. At $d=3$, the following equation, in which $\stackrel{0}{U}, \stackrel{0}{U_{1}}, \stackrel{0}{U}$ and $\stackrel{0}{\mathrm{u}}$ are analytic for $s$ in $V$, holds in any finite number $v$ of sheets (over $V$ ) of the Riemann surface of $\ln \sigma$, for couplings $|\lambda|<\lambda_{0}(v)\left(\lambda_{0}(v)\right.$ tending to zero when $v$ tends to infinity):

$$
F=\stackrel{0}{U}+\frac{\stackrel{0}{U_{1} \cdot{ }_{1}} \stackrel{0}{U}}{a-\stackrel{0}{u}}+\stackrel{1}{F} .
$$

In the latter, the kinematical factor $a(s)$ is equal to $[\ln \sigma]^{-1}$ up to an analytic factor $\operatorname{cst} \sqrt{s}$, and $\frac{1}{F}$ is analytic and bounded at $\sigma=0$ in its multisheeted domain.

Remarks. 1) This result on $\stackrel{1}{F}$ can be alternatively derived from the convergence of the Neumann series $\sum \stackrel{1}{B} \circ \stackrel{1}{B} \ldots \circ \stackrel{1}{B}$ of Eq. (21) by using the decomposition $o=\nabla+g(s) *$ and the fact that $\left|B_{\mid}\right|$is bounded by cst $\times|\sigma||\lambda|$.

2) The decompositions above of $F, B, U$ correspond to decompositions into the "partial wave" $l=0$ and the remainder. A more detailed analysis of the (nonholonomic) structure of $F$ at threshold can be made via a further decomposition of the remainder into partial waves $l \geqq 1$ (see in this connection [BI1]).

Conclusions for $\varphi_{4}^{3}$ are those mentioned in Sect. 1: no pole, hence no bound state or resonance or antibound state locally $(a-\hat{u}$ does not vanish in view of the respective signs of $a$ and $\stackrel{0}{u}$ in the physical sheet at $s<4 \mu^{2}$ and of the imaginary part of $\ln \sigma$ elsewhere). In hypothetical even theories with $\stackrel{0}{u} \approx \lambda$ at first order (instead of $-\lambda$ ), a pole corresponding to a 2-particle bound state at $s=\mu_{B}^{2}, \mu_{B}<2 \mu, 2 \mu-\mu_{B}$ of the order of $e^{-\operatorname{cst} /|\lambda|}$ would occur. There is no known even theory of this type, but such 2-particle bound states will be encountered for non-even theories: see Sect. 5.

\section{Non-Even Theories}

5.1. Preliminaries and Results Away from the 2-Particle Threshold. We start from Eqs. (5)-(7) and from the analyticity and boundedness properties of the 2-p.i. kernels $=2$ = $=B$, =2- and $I_{2}$. The latter are shown in the models to be analytic in strips around euclidean space going up to $(3 \mu)^{2}-\varepsilon$ and to satisfy bounds in cst $|\lambda|$ 
and related asymptotic expansions: see $[\mathrm{S}, \mathrm{K} 1]$ for $P(\varphi)_{2}$ models, or (in all cases) $[\mathrm{IM} 1,2]$.

It is known that generically poles of $F_{1}$ will yield [via (6), (7)] corresponding poles on $=(1-$ and $-(1)$-, but that possible poles of $F$ are different: since $H_{2}=-1 /-1$ - a pole of $F_{1}$ at $s=s_{1}$ yields a zero of $H_{2}$ at $s=s_{1}$ cancelling one of the poles of $=\left(1-\right.$ and $-(1)=$. Moreover the pole at $s=s_{1}$ is cancelled, generically, between $F_{1}$ and the second term in the right-hand side of (5): this is seen easily from the factorization of the residue of the pole of $=11=\left[\operatorname{as} \varphi(k, z) \varphi\left(k, z^{\prime}\right)\right]$. This type of result will be rigorously established in Sect. 5.2 for $s$ in $V$.

At weak coupling, Proposition 1 of Sect. 3 and its stronger version of Sect. 4 at $d=4$ [removal of the condition $\operatorname{dist}\left(D, s=4 \mathrm{~m}^{2}\right)>0$ ] apply similarly [for regions $D$ limited to $s=(3 \mu)^{2}-\varepsilon$ in Minkowski space] to $F_{1}$, and in turn via Eq. (6) (since at $\left.d=4, o=\nabla+\frac{1}{2} *\right)$ to $=(1)-$ and $-(1)=$. On the other hand, $\left|I_{1}\right|$ remains bounded by $\operatorname{cst}(D)|\lambda|$ so that at small enough coupling, $H_{2}$ has no pole in $D$ other than that already known at $s=\mu^{2}$. Hence one has:

Proposition 4. Given any bounded compact region $D$ in their 2-sheeted ( $d$ even) or multisheeted ( $d$ odd) domains, whose distance to $s=4 \mu^{2}$ is $>0, \exists \lambda_{0}$ such that $F$ and $\mathrm{H}_{2}$ have in $\mathrm{D}$ one and only one pole, at $s=\mu^{2}$, if $|\lambda|<\lambda_{0}$. The same result holds also at $d=4$ when the condition $\operatorname{dist}\left(D, s=4 \mu^{2}\right)>0$ is removed.

5.2. The Neighborhood of the 2-Particle Threshold (First Method). We first present in a), b) the easy calculations that can be made directly at $d=2,3$. More general equations that can be written in any dimension and from which previous results can also be derived are given in c) (and proved in Appendix 2).

a) Case $d=2$. Equation (24) yields in this case, as in Sect. 3:

$$
F_{1}=U+\frac{U_{11} U}{a-u}
$$

where $U$ is defined in terms of the 2-p.i. BS kernel $B$. In turn, one has:

Proposition 5.

where

$$
\begin{gathered}
H_{2}=-\frac{(a-u) / d}{a-k}, \\
F=K+\frac{K_{||} K}{a-k},
\end{gathered}
$$

$$
\begin{aligned}
& K\left(k, z, z^{\prime}\right)=U\left(k, z, z^{\prime}\right)-\frac{=\mathbb{N}-(k, z)-\mathbb{N})=\left(k, z^{\prime}\right)}{d(s)}, \\
& =(\mathbb{N}-==(2)+U \nabla=2-\text {, } \\
& d=-(2)+-2=\nabla=(\mathbb{N}) \\
& =-(2)+-(2)=\nabla=(2)+-(2)=\nabla U \nabla=(2) \text {, }
\end{aligned}
$$

and $k$ is the mass-shell restriction of $K: k=u-\frac{n^{2}}{d}, n(s)={ }_{1}=(\mathbb{N}-$ - The kernels $N, d$, and $K$ are analytic at small coupling for $s$ in $V, N$ and $d$ being moreover different from 
zero (and equal to lowest order in $\lambda$ to =-2)- or -(2)=, and -(2)-respectively; in particular d remains close to $3 \mu^{2}$ ).

Proof. Using $o=\nabla+g *, g A * B=\frac{1}{a} A_{||} B$, we first have:

$$
=(1)-(k, z)==(\mathbb{N})-(k, z)+\frac{U_{1}(k, z) n(s)}{a(s)-u(s)} \text {. }
$$

[To see this, write e.g. from (6) and (25):

$$
\begin{aligned}
=(1)= & =(2)-U \nabla=(2)+\frac{1}{a} U_{11}=(2)- \\
& +\frac{U_{1}}{a-u}\left({ }_{1} U \nabla=(2)+\frac{1}{a} u_{1}=(2)-\right)
\end{aligned}
$$

and use $u=(u-a)+a$ in the last term in the right-hand side.]

In a similar way, via (7)

$$
-1)=d+\frac{n^{2}}{a-u}
$$

Equation (26) follows from (31) (since $\left.H_{2}=-[-1)-\right]^{-1}$ ). To obtain (27), write in turn, from (5), (30), (26):

$$
F=U+\frac{U_{11} U}{a-u}+\left(=\mathbb{N}-+\frac{U_{1} n}{a-u}\right)\left(-\mathbb{N}=+\frac{n_{1} U}{a-u}\right) \frac{a-u}{d(a-k)} .
$$

Terms having a pole when $a-u=0$ add up to:

$$
\frac{U_{11} U}{a-u}\left[1+\frac{n^{2}}{d(a-k)}\right]=\frac{U_{11} U}{a-u} \frac{d(a-u)}{d(a-k)}=\frac{U_{11} U}{a-k},
$$

and (27) is in turn obtained [writing e.g. $a-u$, in the term $=(\mathbb{N}-\times-\mathbb{N}=$ $\times(a-u) / d(a-k)$, as $\left.a-k-n^{2} / d\right]$.

In all the formulae, the analyticity of $N, d$, and $K$ follows from the properties of $\nabla$ (Lemma 1).

b) Case $d=3$. The terms $=$ (1) $-(1)=H_{2}$ do not depend on the angle $\theta$ and the term $=\left(1-H_{2}-1\right)=$ in the right-hand side of (5) thus belongs entirely to the $l=0$ component of $F . \stackrel{0}{F}_{1},=(1)-H_{2}$, and $\stackrel{0}{F}$ are thus given by the same formulae as at $d=2$ with $U$ and $K$ replaced by $\stackrel{0}{U}$ and $\stackrel{0}{K}(N \equiv \stackrel{0}{N})$ and $a(s)$ replaced as in Sect. 4 by the adequate factor at $d=3 . F$ is the sum of $\stackrel{0}{F}$ plus the bounded term $\stackrel{1}{F} \equiv \stackrel{1}{F}_{1}$ (treated as in Sect. 4):

\section{Proposition 6.}

$$
\begin{aligned}
H_{2} & =-\frac{(a-\stackrel{0}{u}) / d}{0} \\
a-\stackrel{0}{k} & \\
F & =\stackrel{0}{K}+\frac{\stackrel{0}{K} \stackrel{0}{K}}{a-k}+\stackrel{1}{F}_{1} .
\end{aligned}
$$


Remark. Expressions like $U \nabla=$ (2)- can be written also as $\stackrel{0}{U} \nabla=2$ - (since $\stackrel{1}{U} \nabla=(2)-=0,=(2)-$ being independent of $\theta$ ).

c) General Equations.

\section{Lemma 5.}

$$
\begin{aligned}
& =\left(1-==\mathbb{N}-+F_{1} \hat{*}=(\mathbb{N}-,\right. \\
& F_{1} \hat{*}==\mathbb{N}-=U \hat{*}-(\mathbb{N}), \\
& -(1)=d+-(1)=\hat{*}=(\mathbb{N}-, \\
& F=K+F \hat{*} K=K+K \hat{*} F,
\end{aligned}
$$

where, in Lemma 5, $\hat{*}=g *$, and $N, d, K$ are defined in any dimension by Eqs. (29), $\left(29^{\prime}\right),(28)$.

The proof is given in Appendix 2.

5.3. The Modified or Direct Bethe-Salpeter Kernel and Related Results. The modified or direct BS kernel $G$ defined by Eq. (8) is analytic as =-2-, - (2) = - (2)-at small coupling for $s<9 \mu^{2}-\varepsilon$, if - (2) $\neq 0$ : this is the case at small coupling away from a given neighborhood of $s=\mu^{2}$. [In view of its definition, e.g. - (2) $=s$ $-m^{2}+I_{2}$ in $P(\varphi)_{2},\left|I_{2}\right|<\operatorname{cst}|\lambda|$, - (2) has at $s<(3 \mu)^{2}-\varepsilon$, only one pole at $s=\mu^{\prime 2}, \mu^{\prime}$ close to $\mu$.] In particular, $G$ is analytic at small coupling for $s$ in $V$ and is bounded there by $\operatorname{cst}|\lambda|$.

On the other hand:

\section{Proposition 7.}

$$
F=G+F \circ G
$$

Proof. One has to check that

$$
\begin{gathered}
=(1)=-\frac{=(1--(1)=}{-(1)}-\left(=(2)=-\frac{=(2--(2)}{-(2)}\right)=\left(=(1)=-\frac{=(1)-(1)=}{-(1)}\right) \\
\circ\left(=(2)=-\frac{=(2--(2)=}{-(2)}\right) .
\end{gathered}
$$

This is easily seen by expanding the right-hand side as a sum of 4 terms and using $=(1)=-=(2)==(1)=(2)=(1)=(2)=-(1)-=(2), \quad-(1)=(2)=-(1)=-(2)=$, -(1) $=$ (2) $-=-(1)--(2)$.

Remark. Equations (8), (40) also follow from suitable regroupings of terms in the graphical expansion of $F$, convergent at small coupling in e.g. euclidean space, obtained from [IM1,2]: $G$ is then defined as the sum of graphs that cannot be divided into two parts by cutting 2 lines, with respect to the $2 \rightarrow 2$ channel considered.

Local Structure of F. From (40), one obtains in turn as in Sect. 3:

$$
F=K+g(s) F * K,
$$


where $K$ is defined for $s$ in $V$ in terms of $G$ via the equation

$$
K=G+K \nabla G,
$$

and is, at small coupling, locally analytic as $G$ in $V$ (and equal to $G$ at lowest order in $\lambda$ ).

Remark. The fact that the kernel $K$ thus defined coincides with that of Sect. 5.2 follows from the identity of (41) and (39). Alternatively, one checks directly that (42) is satisfied with $K=U-\frac{=(\mathbb{N})-(\mathbb{N})=}{d}$ and $G$ replaced by its definition (8) [same algebraic proof as that of (40) using here $U \nabla=(2)==U-=(2)=, U \nabla=(2)==(\mathbb{N}-$ $-=(2),-(\mathbb{N})=\nabla=(2)=-(\mathbb{N}=--(2)=$ and $-(\mathbb{N}=\nabla=(2)-d--(2)-]$.

The results on $F$ already mentioned in Sect. 5.2 for $s$ in $V$, such as (27) at $d=2$ or its analogue at $d=3$ follow in this approach from (41).

Local Structure of the 2-Point Function $\mathrm{H}_{2}$. Concerning $\mathrm{H}_{2}$, results can be derived from those on $F$ by the formula:

$$
H_{2}=-\frac{1}{-(2)}+\frac{-(2)=(2-+-(2)=(\mathrm{E})=(2)-}{(-(2)-)^{2}},
$$

easily checked from (5)-(7) and $H_{2}=-1 /$-(1)- .

Proof. From (5), - (2) $\mathrm{F}=(2)=-(2)=(1)=-(-2)=(1)-)^{2} /-(1)-$. Then write -(2) $=(2)-$ - (2) $=(1)=-(2)=(1)-(1)-(2)$.

Remark. Equation (43) also follows graphically from the expansion (convergent at small coupling in euclidean space) of $\mathrm{H}_{2}$ in terms of the 2-p.i. kernels $\mathrm{I}_{2}$ and -2)=. E.g. in $P(\varphi)_{2}$ :

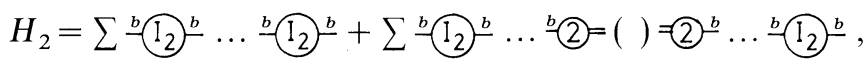

where $\frac{b}{-}$ denotes the bare propagator and where $\sum^{b}-\left(I_{2}\right)^{b} \ldots{ }^{b}-\left(I_{2}\right)^{b}$ is equal to -1 -2) (by definition of -(2) -

Equation (43) gives for $s$ in $V$ (using $o=\nabla+\hat{*}$ )

$$
H_{2}=-\frac{1}{-(2)-}+\frac{-(D)=\hat{*}=(1)-+-(1)=\hat{*} F \hat{*}=(1)-+-(2)=\nabla=(1)-}{(-(2)-)^{2}} \text {, }
$$

where

$$
=(1)-==(2)+K \nabla=(2) \text {. }
$$

In turn (independently of the dimension):

where

$$
\text { (-2) }-)^{2} H_{2}=f+\frac{l^{2}}{a-k} \text {, }
$$

$$
\begin{gathered}
f=f(s)=--(2)+-(2)=\nabla=(1)- \\
=--(2)+-(2)=\nabla=(2)+-(2)=\nabla K \nabla=(2)- \\
l=l(s)=1=(1)-.
\end{gathered}
$$


In all the expressions, $L, l($ as $K, k)$ are locally analytic in neighborhood of $\sigma=0$ in view of Lemma 1. We summarize results on $\mathrm{F}$ and $\mathrm{H}_{2}$ in:

Proposition 8.

$$
\begin{gathered}
F=K+\frac{K_{||} K}{a-k}, \\
H_{2}=f^{\prime}+\frac{l^{2}}{a-k},
\end{gathered}
$$

where $f^{\prime}, l^{\prime}, k$ are analytic for $s$ in $V$ (at small coupling).

In (49), $f^{\prime}=f /(-(2)-)^{2}, l^{\prime}=l /$-(2) - and, as already mentioned, -(2)- $=3 \mu^{2}$ $+O(\lambda)(\neq 0)$.

The coïncidence of the expressions (49) and (26) of $\mathrm{H}_{2}$ is checked directly in Appendix 2.

5.4. Applications. Results on poles and residues are obtained as in Sect. 4 with $U$ replaced by $K$ (itself equal to $G$ at lowest order in $\lambda$ ).

In $P(\varphi)_{2}$ models, one finds again, according to the model, one pole in the physical or second sheet.

In a model like e.g. $\left(\lambda \varphi^{4}+\lambda^{\prime} \varphi^{3}\right)$ in dimension 2 or 3 (or models that reduce to it such as those mentioned in Sect. 1), the kernel $k$ is equal at lowest order in $\lambda, \lambda^{\prime}$ and at $\sigma=0$, to a sum of contributions of the form $-\lambda$ (graph $><$ )

$$
\left.\lambda^{\prime 2} / \mu^{2} \text { (graphs of the form }{ }^{1} Y: p_{1}=\ldots=p_{4} \text { at } \sigma=0\right)
$$

and $-\frac{\lambda^{\prime 2}}{3 \mu^{2}}\left(\right.$ graphs $\frac{1}{2}><$ in = (2)- $H_{2}^{\prime}-(2), H_{2}^{\prime}=-\frac{1}{-(2)-}=-\frac{1}{3 \mu^{2}}$ at lowest order in $\left.\lambda, \lambda^{\prime},=2-\approx \lambda^{\prime}\right)$. There will be one pole in the physical sheet (hence a 2-particle bound state) at $s=\mu_{B}^{2}, \mu_{B}<2 \mu$ if the sum is $>0$. This depends on the relative values of $\lambda$ and $\lambda^{\prime}$, e.g. on the constant $\alpha$ if $\lambda^{\prime 2}=\alpha \lambda$. In this case, $\left|\mu_{B}-2 \mu\right|$ behaves at $d=3$, as already mentioned in Sect. 1 , as $e^{- \text {cst } / \lambda}$ as $\lambda \rightarrow 0$. If $\alpha$ is such that the sum is $<0$, there is one pole in the second sheet at $d=2$ and no pole (for $s$ in $V$ ) at $d=3$. If $\alpha$ is such that the sum is zero, a calculation to next order is needed.

\section{Appendix 1: Results on 2-Particle Convolution}

This Appendix gives the proof of Lemma 1 of Sect. 4 according to which the 2-particle Feynman-type convolution $o$ is regular at the 2-particle threshold after extraction of $g(s) *$.

Lemma 1 will be established in Sect. b) for $d=2$ and $d=3$ by a procedure that makes use of known results on the Feynman integral $\bigcirc$ and of a simple geometrical idea (presented in detail and extensively applied in [BI 2]). We first outline in Sect. a) an alternative proof based on geometrical properties of the contour $\Gamma(k)$, to which the analysis of simple quadratic pinch situations of $[\mathrm{L}, \mathrm{Ph}]$ is applicable; the latter is valid for any dimension $d$. 
a) Geometrical Proof. It is known from the outset that $A \nabla B$ can be analytically continued, as $A \circ B$ and $g(s) A * B$, in a two-sheeted ( $d$ even) or multisheeted ( $d$ odd) domain around $\sigma=0$. The fact that $A \nabla B$ is uniform when one turns around $\sigma=0$ is, as already indicated in [Ia], a consequence of the relation (see $[\mathrm{Ph}, \mathrm{BP}])^{3}$ :

$$
O_{+}-o_{-}=*_{+},
$$

where $o_{+}$and $o_{-}$are the restrictions of $o$ to values of $k$ in the region $s>4 \mu^{2}$ obtained respectively from the sides $\operatorname{Im} s>0$ and $\operatorname{Im} s<0$ of the physical sheet [and correspond to integration contours $\left.\Gamma_{+}(k), \Gamma_{-}(k)\right]$. In fact, $(g(s) *)_{+}-(g(s) *)_{-}$is also equal, by direct inspection in each parity case, to $*_{+}$so that $\nabla_{+}-\nabla_{-}=0$.

The proof of Lemma 1 can be completed from more refined geometrical results of $[\mathrm{Ph}]$ (see also [BP]). At $d$ even, $\Gamma(k)$ can be written as:

$$
\Gamma(k)=\underline{\Gamma}+\frac{1}{2} \tilde{e}(k),
$$

where $\underline{\Gamma}$ is a fixed cycle independent of $k$ whose support stays at a fixed $(>0)$ distance of the poles of 2-point functions and $\tilde{e}(k)$ is a cycle whose support surrounds the mass-shell, such that integration over $\tilde{e}(k)$ reduces to $*$ by a residue formula. From its definition $\left(2^{\prime}\right), \nabla$ thus amounts to integration over $\underline{\Gamma}$ and Lemma 1 follows in a straightforward way from the fact that the support of $\underline{\Gamma}$ is not pinched as $s \rightarrow 4 \mu^{2}$.

At $d$ odd, a decomposition of $\Gamma(k)$ into three parts given by [Ph], which is more complicated and does not provide a simple expression for $\nabla$, is obtained. It can be used to show that $|A \circ B|<\operatorname{cst}|\ln | \sigma||$ (as in $[\mathrm{Ph}]$ and [BP]), and therefore that $|A \nabla B|\left(\right.$ where $\nabla$ is defined as $\left.o-\frac{i \ln \sigma}{2 \pi} *\right)$ is bounded by cst $|\ln | \sigma||$. The uniformity of $A \nabla B$ then yields its local analyticity for $s$ in $V$ and Lemma 1 . Note that a more explicit geometrical characterization of $\nabla$ will be given in [BI2] and will allow a simpler and more satisfactory presentation of the argument.

b) Proof Making Use of Results on the Feynman Integral for $d=2,3$. We below evaluate $A \circ B$ as follows. It will be assumed, as is the case in the application in view of Lorentz invariance, that the dependence of $A(k, z, \zeta)$ or $B\left(k, \zeta, z^{\prime}\right)$ with respect to $\zeta$ reduces to a dependence on $\zeta_{0}, \zeta_{1}^{2}$ at $d=2$, or at $d=3$ on $\zeta_{0}, \zeta^{2}$ and supplementary angle variables [angle between $\mathbf{z}$ and $\zeta$, or $\zeta$ and $\mathbf{z}^{\prime} ; k$ is taken of the form $\left.\left(k_{0}, \mathbf{0}\right)\right]$. By definition:

$$
A \circ B\left(k, z, z^{\prime}\right)=\int A(k, z, \zeta) \omega(k, \zeta) B\left(k, \zeta, z^{\prime}\right) d \zeta,
$$

where $\omega$ can also be written as:

$$
\omega(k, \zeta)=\frac{1}{((k / 2)+\zeta)^{2}-\mu^{2}} \frac{1}{((k / 2)-\zeta)^{2}-\mu^{2}} \alpha(k, \zeta)
$$

\footnotetext{
${ }^{3}$ This relation corresponds to a special case of the Picard-Lefschetz formula, which expresses the variation of the integration cycle around the singularity created by a pinch situation. In the present case, it can be proven directly, e.g. by the argument given in the course of the proof of Theorem 1 of [BL] [derivation of the third term of the discontinuity formula (2), given in paragraph 2.4.3]; another simple proof of it will be found in [BI2]
} 
with $\alpha(k, \zeta)$ analytic and bounded in $\Delta \times \mathscr{D}$.

A will then be decomposed into $A_{1}+A^{\prime}$, where

$$
A_{\mid}(k, z, \zeta)=A\left(k, z, \zeta_{0}=0, \zeta^{2}=-\frac{\sigma}{4}, \theta\right)
$$

$\zeta_{0}=0, \zeta^{2}=-\frac{\sigma}{4}$ being the values that make the intermediate momenta $k_{ \pm}=\frac{k}{2} \pm \zeta$ on-shell: $k_{ \pm}^{2}-\mu^{2}=0 . B\left(k, \zeta, z^{\prime}\right)$ is decomposed similarly as $\beta B+B^{\prime}$, as also $\alpha(k, \zeta)$ $\left(=\underline{\alpha}\left(k,\left(0,-\frac{\sigma}{4}\right)\right)+\alpha^{\prime}\right)$.

The basic geometrical idea to be used below is the following. The differences $A^{\prime}=A-A_{1}, B^{\prime}=B-{ }_{1} B$ or $\alpha^{\prime}=\alpha-\underline{\alpha}$ generate factors that will cancel one of the poles produced by the Feynman propagators: it is then easily seen that the integration contour in corresponding contributions is no longer pinched and can thus be moved so as to avoid the remaining pole singularity. Such contributions will therefore be analytic in the neighborhood of $\sigma=0$.

More precisely, we separate contributions to the integral (A.3) into two parts:

(i) Those corresponding to the terms $A_{1,} B$, $\underline{\alpha}$, which do not depend on $\zeta_{0}, \zeta^{2}$.

The Feynman integral

$$
I(k)=\int \frac{1}{((k / 2)+\zeta)^{2}-\mu^{2}} \times \frac{1}{((k / 2)-\zeta)^{2}-\mu^{2}} d \zeta,
$$

is known explicitly (as will be admitted here) and can be analytically continued when $k$ is taken away from euclidean space. It has a singularity in $\sigma^{-1 / 2}$ or $\ln \sigma$ in the complex neighborhood of $\sigma=0$ and the explicit straightforward evaluation then shows, in view of the definition of $*$, that:

$$
\int \frac{A_{||} B \underline{\alpha} d \zeta}{\left[((k / 2)+\zeta)^{2}-\mu^{2}\right]\left[((k / 2)-\zeta)^{2}-\mu^{2}\right]}=g(s)(A * B)+C,
$$

where the term $C$ is analytic for $s$ in $V$ and satisfies the properties required in Lemma 1.

(ii) All other terms which will be evaluated by dividing the integration contour into a local part conveniently chosen and the remainder. The latter is treated by separating all terms that occur in the differences $A-A_{1}, B-{ }_{1} B$ or $\alpha-\underline{\alpha}$. It is checked that all contributions can be analytically continued with no singularity at $s=4 \mu^{2}$ (the factors $k_{ \pm}^{2}-\mu^{2}=-\frac{\sigma}{4}+\zeta^{2} \pm k_{0} \zeta_{0}$ do not vanish).

Finally, the local part is treated by noting that the differences generate factors $\zeta_{0}$ or $\zeta^{2}+\frac{\sigma}{4}$ equal respectively to $\frac{1}{k_{0}}\left[\left(k_{+}^{2}-\mu^{2}\right)-\left(k_{-}^{2}-\mu^{2}\right)\right]$ or $\frac{1}{2}\left[\left(k_{+}^{2}-\mu^{2}\right)\right.$ $\left.+\left(k_{-}^{2}-\mu^{2}\right)\right]$, so that this part is equal to a sum of terms in which one of the two Feynman propagators has been cancelled. This is seen also by going from the variables $\zeta_{0},|\zeta|$ to $s_{+}, s_{-}, s_{ \pm}=\left(\frac{k}{2} \pm \zeta\right)^{2}-\mu^{2}$. The geometrical idea described above thus applies to this part. 
Analyticity of $A \nabla B$, once $g(s) A * B$ has been subtracted from $A \circ B$, is thus established and the bounds of Lemma 1 are easily obtained for all relevant contributions.

\section{Appendix 2: Technical Complements (Non-Even Theories)}

a) Proof of Lemma 5. Equation (36) is obtained e.g. by writing

$$
\begin{aligned}
=(1)=\hat{*}=(\mathbb{1})- & =(1)=\hat{*}(=(2)+U \nabla=(2)-) \\
& ==(1)=\hat{*}=(2)+(=(1)=\hat{*} U) \nabla=(2)- \\
& ==(1)-=(2--U \nabla=(2) \equiv=(1)-=\mathbb{N}-
\end{aligned}
$$

(where we have used $=(1)=\hat{*} U==(1)=-U, o=\nabla+\hat{*}$ ). Equations (37) and (38) are also obtained easily. Finally, to prove (39), one has to check that

$$
\begin{aligned}
=(1)= & -\frac{=(1)-(1)=}{-(1)-}-\left(U-\frac{=(\mathbb{N})-(\mathbb{N})=}{d}\right) \\
& =\left(=(1)-\frac{=(1--(1)=}{-(1)}\right) \hat{-}\left(U-\frac{=(\mathbb{N}--(\mathbb{N})=}{d}\right) .
\end{aligned}
$$

This is achieved by expanding the right-hand side as a sum of 4 terms and using $=(1)=-U==(1)=\hat{*} U$, as also (36) $-(38)$.

b) Direct check of the coincidence of the expression (26) and (49) of $\mathrm{H}_{2}$. Equation (26) will be written (using $a-u=a-k-n^{2} / d$ ) in the form:

$$
H_{2}=-\frac{1}{d}+\frac{n^{2} / d^{2}}{a-k}
$$

In (45), $L$ (and in turn $f, l$ ) are defined in terms of $K$ by Eq. (44'). Replacing $K$ by its expression $U-=\mathbb{N}--\mathbb{N}=/ d$, one has:

$$
\begin{aligned}
=(1)- & ==(2)-U \nabla=(2)--\frac{=(\mathbb{N}--\mathbb{N})=\nabla=(2)-}{d} \\
& ==\mathbb{N}-\left(1-\frac{N \nabla=(2)-}{d}\right) \\
& ==\mathbb{N}-\frac{-(2)}{d}(\text { since } N \nabla=(2)-d--(2)-) .
\end{aligned}
$$

Hence $l^{2} /(-2-)^{2}=n^{2} / d^{2}$ and:

$$
f=--(2)--(2)=\nabla=\left(\mathbb{N}-\frac{-(2)}{d}=-(-2)-\right)^{2} / d
$$

where we have used again $-(2)=\nabla=\mathbb{N}-=d-$-(2) . 


\section{References}

[B1] Bros, J.: In: Analytic methods in mathematical physics, p. 85. New York: Gordon and Breach 1970

[B2] Bros, J.: Contribution to the book "Prospect of Algebraic Analysis" (dedicated to M. Sato), to be published, Saclay preprint SPhT 87-154

[BI1] Bros, J., Iagolnitzer, D.: Commun. Math. Phys. 85, 197 (1982)

[BI2] Bros, J., Iagolnitzer, D.: In preparation

[BL] Bros, J., Lassalle, M.: Commun. Math. Phys. 43, 279 (1975); 54, 33 (1977)

[BP] Bros, J., Pesenti, D.: J. Maths. Pures Appl. 58, 375 (1980)

[DE1] Dimock, J., Eckmann, J.-P.: Commun. Math. Phys. 51, 41 (1976)

[DE2] Dimock, J., Eckmann, J.-P.: Ann. Phys. 103, 289 (1977)

[EE] Eckmann, J.-P., Epstein, H.: Commun. Math. Phys. 68, 265 (1979)

[FMRS] Feldman, J., Magnen, J., Rivasseau, V., Sénéor, R.: Commun. Math. Phys. 103, 67 (1986)

[GJ] Glimm, J., Jaffe, A.: Commun. Math. Phys. 67, 267 (1979)

[GJS] Glimm, J., Jaffe, A., Spencer, T.: Ann. Math. 10, 585 (1974) and Lecture Notes in Physics, Vol. 39. Araki, H. (ed.). Berlin, Heidelberg, New York: Springer 1975

[GK] Gawedzki, K., Kupiainen, A.: Commun. Math. Phys. 99, 197 (1985)

[H] Heitler, W.: Proc. Camb. Phil. Soc. 37, 291 (1941)

[Ia] Iagolnitzer, D.: Commun. Math. Phys. 88, 235 (1983)

[IM1] Iagolnitzer, D., Magnen, J.: Commun. Math. Phys. 110, 51 (1987)

[IM2] Iagolnitzer, D., Magnen, J.: Commun. Math. Phys. 111, 81 (1987)

[Im] Imbrie, J.Z:: Commun. Math. Phys. 78, 169 (1980)

[K 1] Koch, H.: Ann. Inst. Henri Poincaré 31, 1979, p. 173

[K 2] Koch, H.: Helv. Phys. Acta 53, 429 (1980)

[L] Leray, J.: Bull. Soc. Math. de France 87, 81 (1959)

[Ph] Pham, F.: Introduction a l'Etude Topologique des Singularités de Landau, Memorial des Sciences Mathématiques, Vol. 164. Paris: Gauthier-Villars 1967

[POLE] Eden, R.J., Landshoff, P.V., Olive, D.I., Polkinghorne, J.C.: The analytic $S$ Matrix. Cambridge: Cambridge University Press 1966

[S] Spencer, T.: Commun. Math. Phys. 44, 153 (1975)

[SZ] Spencer, T., Zirilli, F.: Commun. Math. Phys. 49, 1 (1976)

[Z] Zimmerman, W.: Nuovo. Cimento 21, 429 (1961)

Communicated by K. Gawedzki

Received April 26, 1988 
